\title{
Anisotropic cosmological models with spinor field and viscous fluid in presence of a $\Lambda$ term: qualitative solutions
}

\author{
Bijan Saha and Victor Rikhvitsky \\ Laboratory of Information Technologies \\ Joint Institute for Nuclear Research, Dubna \\ 141980 Dubna, Moscow region, Russid*
}

(Dated: October 25, 2018)

\begin{abstract}
The study of a self-consistent system of nonlinear spinor and Bianchi type I gravitational fields in presence of a viscous fluid and $\Lambda$ term with the spinor field nonlinearity being some arbitrary functions of the invariants $I$ an $J$ constructed from bilinear spinor forms $S$ and $P$, generates a multi-parametric system of ordinary differential equations [1, 2]. A qualitative analysis of the system in question has been thoroughly carried out. A complete qualitative classification of the mode of evolution of the universe given by the corresponding dynamic system has been illustrated.
\end{abstract}

PACS numbers: 03.65.Pm and 04.20.Ha

Keywords: Spinor field, Bianchi type I (BI) model, Cosmological constant, viscous fluid, qualitative analysis

\section{INTRODUCTION}

Though the investigation of relativistic cosmological models usually has the energy momentum tensor of matter generated by a perfect fluid, to consider more realistic models one must take into account the viscosity mechanisms $[3,4,5,6,6,7]$. On the other hand in the recent years anisotropic cosmological models with nonlinear spinor field have been extensively studied due to the facts that (i) introduction of nonlinear spinor field into the system leads to the isotropization of initially anisotropic universe [8, 9, 10]; (ii) spinor field nonlinearity in some cases can give rise to singularity-free solutions [10, 11]; (iii) spinor field can be considered as one of the possible candidate to explain the late time acceleration of the universe [12, 13].

Given the importance of viscous fluid and spinor field to model a realistic universe, recently we have considered a self-consistent system of nonlinear spinor field and a gravitational field described by a Bianchi type I (BI) cosmological model filled with viscous fluid [1, 2]. In [1, 2] we have thoroughly studied the corresponding field equations. Exact solutions of the field equations were given in terms of volume scale of the BI space-time $\tau$. Multi-parametric system of equations for volume scale $\tau$, energy density of the viscous fluid $\varepsilon$ was solved for some special choice of bulk and share viscosity. Given the richness of the system mentioned above in this report we study it qualitatively for more general cases. In the absence of viscosity the system allows integrals of motion, whereas it is either impossible obtain, or there is no first integral at all in general when the viscosity is taken into account. As a result the study of possible modes of evolutions becomes very difficult. Undoubtedly, the result of the investigation should be presented in the form of numerical values and at the same time can not be reduced to a representation of some causally

*Electronic address: bijan@jinr.ru; URL: http://www.jinr.ru/ bijan/ 
found examples of numerical solutions. Clearly, it should be a classification of modes of the evolution in the parametric space. Actually, it is the task of qualitative analysis.

\section{BASIC EQUATIONS}

We consider a self-consistent system of nonlinear spinor and Bianchi type-I (BI) gravitational fields filled with a viscous fluid in presence of a cosmological term. As it was shown in [1, 2], the components of the spinor field and metric functions can be expressed in terms of volume scale $\tau$ of the BI space-time. So one needs to find the function $\tau$, explicitly. Corresponding equation can be derived from Einstein equations and Bianchi identity [a detailed description of this procedure can be found in [1, 2]]. For convenience, we also define the generalized Hubble constant. The system then reads:

$$
\begin{aligned}
\dot{\tau} & =3 H \tau \\
\dot{H} & =\frac{1}{2}(3 \xi H-\omega)-\left(3 H^{2}-\kappa \varepsilon-\Lambda\right)+\frac{\kappa}{2}\left(\frac{m}{\tau}+\frac{\lambda(n-2)}{\tau^{n}}\right), \\
\dot{\varepsilon} & =3 H(3 \xi H-\omega)+4 \eta\left(3 H^{2}-\kappa \varepsilon-\Lambda\right)-4 \eta \kappa\left[\frac{m}{\tau}-\frac{\lambda}{\tau^{n}}\right] .
\end{aligned}
$$

Here $\kappa$ is the Einstein's gravitational constant, $\Lambda$ is the cosmological constant, $\lambda$ is the selfcoupling constant, $m$ is the spinor mass and $n$ is the power of nonlinearity of the spinor field (here we consider only power law nonlinearity). In (2.1) $\eta$ and $\xi$ are the bulk and shear viscosity, respectively and they are both positively definite, i.e.,

$$
\eta>0, \quad \xi>0
$$

They may be either constant or function of time or energy. We consider the case when

$$
\eta=A \varepsilon^{\alpha}, \quad \xi=B \varepsilon^{\beta}
$$

with $A$ and $B$ being some positive quantities. For $p$ we set as in perfect fluid,

$$
p=\zeta \varepsilon, \quad \zeta \in(0,1]
$$

Note that in this case $\zeta \neq 0$, since for dust pressure, hence temperature is zero, that results in vanishing viscosity. Note that a system in absence of spinor field has been studied in [14, 15]. In that case the corresponding system is analogical to the one given in (2.1) without the third terms in (2.1b) and (2.1c).

\section{QUALITATIVE ANALYSIS}

Research on the behavior of the dynamic system given by a system of ordinary differential equations implies the survey of all possible scenarios of development for different values of the problem parameters. It is necessary to understand at least how the process of evolution comes to an end if it does so at infinitively large time for a given set of initial conditions which can be given anywhere.

So, under the specific behavior of the system we understand the phase portrait of the system, i.e., the family of integral curves, covering the total phase space. It is easy to imagine as far as 
any point of the space can be declared as the initial one and at least one integral curve will pass through it (or it will be fixed point).

Certainly, it is difficult to imagine such a set of curves. In many cases, close (and not only) curves transform into each other at some diffeomorphism of space. These curves are known as topologically equivalent. The differences between them are not very important for our study. They all behave in the same manner. This relation - "the relation of equivalence" - divides the family of curves into the classes of equivalence. For graphical demonstration it will be convenient to present at least one representative of each class.

The change of the value of problem parameters not always results in significant change of the phase portrait. Repeating this method, we say that one family of integral curves (covering the total space) for the given set of parameters is equivalent to the other for another set of parameters, if there exists a diffeomorphism of space transforming the first family into the second. It is clear that there occurs the division into the classes of equivalence, and we are not very interested in differences between equivalent families. We argue that the corresponding changes in parameters do not alter anything on principle. So it is sufficient to demonstrate only one phase portrait for a given set of parameters underlining the features of the given class.

However, for some critical relations between the parameters there occurs significant changes. These are the boundary relations of parameters, dividing, as usual, parameter space into regions of similar behavior. Thus accomplishes the qualitative classification of the mode of evolution of dynamic system. Now, giving the concrete value of parameters, we can define which region of parameters they correspond to, thus define the type of behavior. Moreover, given the specific initial conditions, we can answer the question to which region of phase space the evolution of the system lead in time.

In our cosmological model, numerical parameters $A, \alpha, B, \beta$ are related to the viscosity, while $\lambda$ and $\Lambda$ are the (self)-coupling and cosmological constants.

Initially, we consider the system of Einstein and Dirac equations. Solving these equations, we find the components of the spinor field and metric functions $a, b, c$ in terms of volume scale $\tau=a b c$ of the BI universe. Finally, in order to find $\tau$ from Einstein equations and Bianchi identity, we deduce three first order ordinary differential equations. Further for convenience we introduce a new function $v$ inverse to $\tau$, i.e., $v=1 / \tau$.

The fact that the system has the dimension greater than 2 , strongly complicates qualitative analysis. Note that well known Lorentz system of three ordinary differential equations with polynomial right hand side with degree less or equal to 2 , possesses in some region of parameter space chaotic behavior known as a strange attractor and in that region there do not exist first integrals (i.e., globally defined invariants). Though the set of singularities is very simple, there exist only three singular (fixed) points: two focus and one saddle. The presence of such example does not allow us to make an optimistic conclusion on the basis of simple construction of our system (with polynomials in the right hand side and absence of singular points the in region of space we are interest in, which is even dynamically closed.

Nevertheless, on the boundary of the the space $\varepsilon=0$, as well as $v=0(\tau=+\infty)$, which are dynamically closed themselves, the complete classification has been done. The dynamical closeness of these planes simultaneously as an obstacle for penetration from positive octant $\varepsilon>0$ $\wedge v>0$ to the region with negative values. But, there are no singularities, fixed points (there are fixed points on the boundary) in the positive octant, we were not able to prove the simplicity of its behavior, e.g., presence of first integrals, as well as their absence.

Thus let us go back to the system (2.1) in details. As it was already mentioned, tt is convenient to define a new function $v=1 / \tau$. In this case the obvious singularity that occurs at $\tau=0$ vanishes and $v=0$ corresponds to $\tau=\infty$ while $v=\infty$ to $\tau=0$. The system (2.1) on account of (2.3) takes 
the form:

$$
\begin{aligned}
\dot{v} & =-3 H v \\
\dot{H} & =\frac{1}{2}\left(3 B \varepsilon^{\beta} H-(1+\zeta) \varepsilon\right)-\left(3 H^{2}-\varepsilon-\Lambda\right)+\frac{1}{2}\left(m v+\lambda(n-2) v^{n-1}\right) \\
\dot{\varepsilon} & =3 H\left(3 B \varepsilon^{\beta} H-(1+\zeta) \varepsilon\right)+4 A \varepsilon^{\alpha}\left(3 H^{2}-\varepsilon-\Lambda\right)-4 A \varepsilon^{\alpha}\left[m v-\lambda v^{n}\right] .
\end{aligned}
$$

Let us now study the foregoing system of equations in details.

\section{A. Behavior of the solutions on $v=0$ plane}

Let us first study the behavior of the functions $H$ and $\tau$ on $v=0$ plane. The plane $v=0$ is dynamically invariant, since $\left.\dot{v}\right|_{v=0}=0$. It should be emphasized that the system in this case coincides with one in absence on spinor field and was thoroughly studied in [15]. Nevertheless, we write the results obtained in detail. In doing so we rewrite the Eqs. (3.1b) and (3.1c) in the matrix form:

$$
\left(\begin{array}{c}
\dot{H} \\
\dot{\varepsilon}
\end{array}\right)=\left(\begin{array}{cc}
\kappa / 2 & -1 \\
3 H & 4 \eta
\end{array}\right)\left(\begin{array}{c}
3 B \varepsilon^{\beta} H-(1+\zeta) \varepsilon \\
3 H^{2}-\kappa \varepsilon-\Lambda
\end{array}\right) .
$$

a) By virtu of linear independence of the columns of the matrix of the Eq. (3.2) the critical points are the solutions of the equations

$$
\begin{aligned}
3 B \varepsilon^{\beta} H-(1+\zeta) \varepsilon & =0 \\
3 H^{2}-\kappa \varepsilon-\Lambda & =0
\end{aligned}
$$

i.e., they necessarily lie on the parabola (3.3b). In view of

$$
H=\frac{1+\zeta}{3 B} \varepsilon^{1-\beta}
$$

which follows from (3.3a), the Eq. (3.3b) can be written as

$$
3 \kappa B^{2} \varepsilon^{1+2 \beta}-(1+\zeta)^{2} \varepsilon^{2}+3 \Lambda B^{2} \varepsilon^{2 \beta}=0 .
$$

The solutions to the system (3.3) will be the roots of the Eq. (3.4). The quantity of the positive roots of Eq. (3.4) according to Cartesian law is equal to the number of changes of sign of the coefficients of equations or less than that by an even number. So, for $\Lambda>0$ and $1 / 2<\beta<1$ or $\Lambda<0$ and $\beta<1 / 2$ the number of roots is either 2 or zero. For the remaining cases, i.e., $\Lambda>0$ and $\beta>1$ or $\Lambda>0$ and $\beta<1 / 2$ or $\Lambda<0$ and $\beta>1 / 2$ there exists only one root.

In Table 1 classification of qualitatively different types of evolution (phase portrait) depending on the parameters $\beta, \Lambda$ and $(1+\zeta) / B$ are illustrated. The Fig. a) in Table 1 corresponds to the two types, namely $\beta<1 / 2$ or $\beta=1 / 2$ and $(1+\zeta) / B<\sqrt{3 \kappa}$, as well as the Fig. i) to the cases $\beta>1$ or $\beta=1$ and $(1+\zeta) / B<\sqrt{3 \kappa}$. The Figs. g) and h) of Table 1 cover all the four cases for $\beta>1 / 2$. The Figs. d) and e) contain pairs of graphics (case with 0 or 2 singular points; case with 1 singular point is also allowable since it possesses the frequency 2, i.e., two singular points merges to one saddle-knot and no other qualitative change takes place). They covers 3 cases: $d$ ) corresponds to $\beta \leq 1 / 2$; e) corresponds to $\beta=1 / 2$ and $(1+\zeta) / B<\sqrt{3 \kappa}$ or $1 / 2 \leq \beta \leq 1$ and $\beta=1 / 2$ and and $(1+\zeta) / B>\sqrt{3 \kappa}$. 


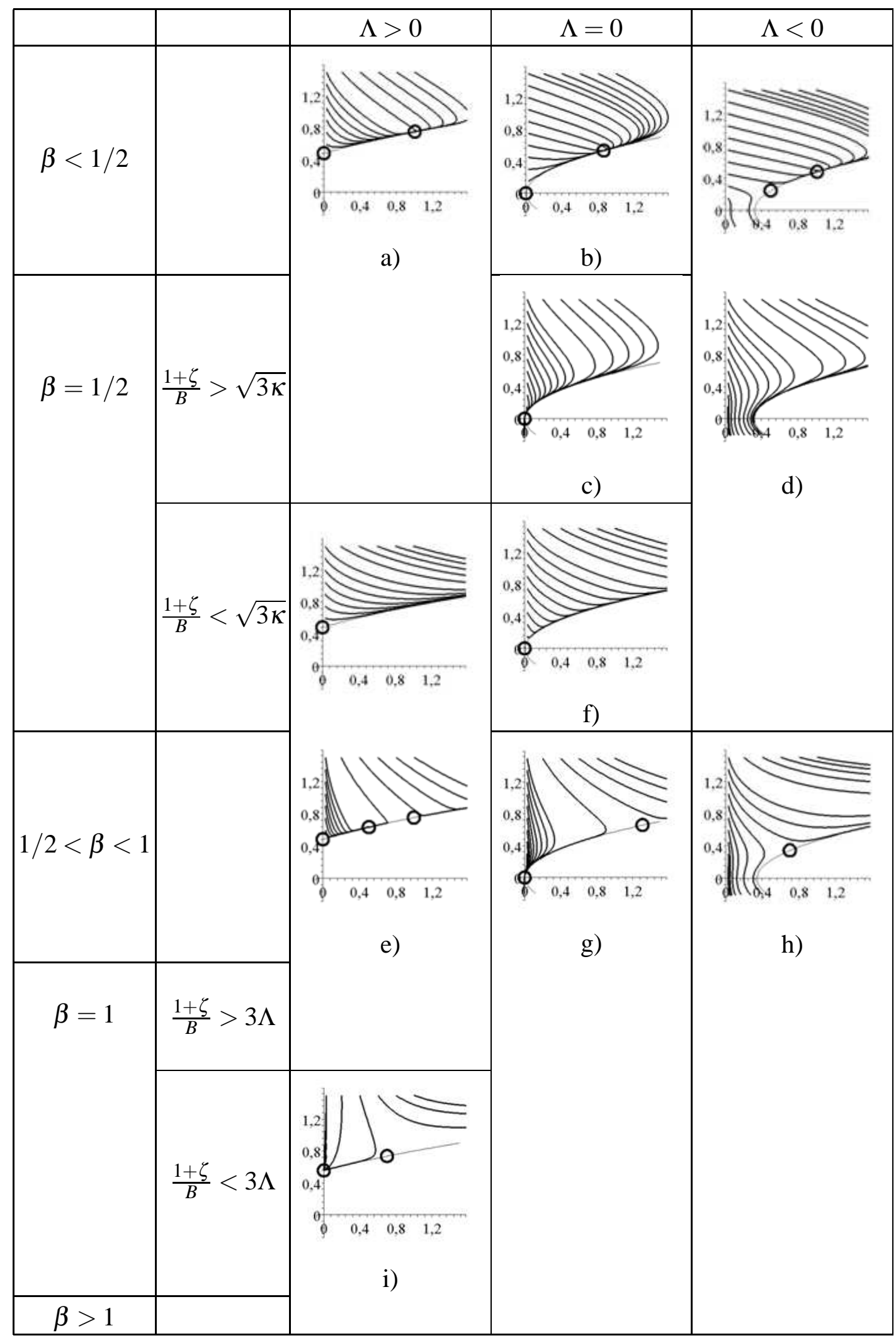

Table 1. Classification of qualitatively different types of evolution (phase portrait) depending on the parameters $\beta, \Lambda$ and $(1+\zeta) / B$ 
Since, the equation for $\varepsilon$ only contains $\eta$, the energy density for nontrivial $\eta$ undergoes essential changes, whereas $H$ and $\tau$ remain virtually unchanged.

The types of critical points lying on the integral curve alternate: ... saddle, attracting knot, saddle .... So it is sufficient to consider the case with maximum number of roots. Taking into account the Eqs. (2.1c) and (3.3b) let us now calculate

$$
\begin{aligned}
\lim _{\varepsilon \rightarrow+\infty} \frac{\dot{\varepsilon}}{3 H \varepsilon} & =\lim _{\varepsilon \rightarrow+\infty} \frac{\sqrt{3} B \varepsilon^{\beta} \sqrt{\kappa \varepsilon+\Lambda}-\varepsilon(1+\zeta)}{\varepsilon} \\
& =\sqrt{3} B \sqrt{\kappa \varepsilon^{(2 \beta-1)}+\Lambda \varepsilon^{-2}}-(1+\zeta)=\left\{\begin{array}{cc}
-(1+\zeta)<0, & \beta<1 / 2, \\
B \sqrt{3 \kappa}-(1+\zeta), & \beta=1 / 2, \\
+\infty>0, & \beta>1 / 2 .
\end{array}\right.
\end{aligned}
$$

So, the latest critical point for $\beta<1 / 2$ is attracting knot and for $\beta>1 / 2$ is saddle. In case of $\beta=1 / 2$ we have either saddle if $B \sqrt{3 \kappa}-(1+\zeta)>0$ and attracting knot otherwise.

b) It is obvious that if $\Lambda \geq 0$ the points of intersection of the boundary are the critical points

$$
\begin{aligned}
H & = \pm \sqrt{\Lambda / 3} \\
\varepsilon & =0
\end{aligned}
$$

c) For $H<0$ there may exist critical points, if the columns of the matrix of (3.2) are linearly dependent. In that case the critical points are the roots of the equation

$$
3 \kappa(\zeta-1) \varepsilon+6 \kappa^{2} A B \varepsilon^{\alpha+\beta}+8 \kappa^{2} A^{2} \varepsilon^{2 \alpha}-6 \Lambda=0
$$

and

$$
H=-\frac{2}{3} \kappa A \varepsilon^{\alpha}
$$

In case of $\eta=0$ the roots of the characteristic equation

$$
\left|\frac{D(\dot{H}, \dot{\varepsilon})}{D(H, \varepsilon)}-\mu\right|=0
$$

are

$$
\mu_{1,2}=\frac{3 \kappa \xi \pm \sqrt{9 \kappa^{2} \xi^{2}+48 \Lambda(1+\zeta)}}{4} .
$$

The critical point $(H, \varepsilon)=(0,2 \Lambda /[\kappa(\zeta-1)])$ is of type divergent focus if $\Lambda>-9 \kappa^{2} \xi^{2} /[48(1+$ $\zeta)]$ or divergent knot if $\Lambda<-9 \kappa^{2} \xi^{2} /[48(1+\zeta)]$.

\section{Integral curves}

For $\Lambda \geq 0$ the solutions starting from the upper half-plane $H>0$ cannot enter into the lower one. For $\Lambda<0$ some of the solutions may enter into the lower half-plane through the segment $H=0$ and $\Lambda \leq 0 \leq \varepsilon$ and never returns back, since $\left.\dot{H}\right|_{H=0}<0$. 


\section{B. Behavior of the solutions on $\varepsilon=0$ plane}

The plane $\varepsilon=0$ is dynamic invariant, since $\left.\dot{\varepsilon}\right|_{\varepsilon=0}=0$. Depending on the sign of $H$ this plane is either attractive or repulsive, namely, for $H>0$ it is attractive and for $H<0$ it is repulsive, since

$$
\frac{\partial \dot{\varepsilon}}{\partial \varepsilon}=-3 H(1+\zeta)<0
$$

On $\varepsilon=0$ plane the system (3.1) takes the form

$$
\begin{aligned}
\dot{v} & =-3 H v \\
\dot{H} & =-3 H^{2}+\Lambda+\frac{1}{2}\left(m v+\lambda(n-2) v^{n-1}\right) .
\end{aligned}
$$

The system (3.11) has the following integrals:

$$
\begin{aligned}
& 3 H^{2}=C v^{2}+m v+\Lambda-\frac{\lambda(n-2) v^{n-1}}{n-3}, \quad n>3, \\
& 3 H^{2}=C v^{2}+m v+\Lambda-v^{2} \ln (v), \quad n=3, \\
& 3 H^{2}=C v^{2}+m v+\Lambda, \quad n=2,
\end{aligned}
$$

where $C$ is some arbitrary constant.

The characteristic equation of nontrivial singular points on $\varepsilon=0$ plane for the system (2.1) takes the form

$$
\lambda(n-2) v^{n-1}+m v+2 \Lambda=0 .
$$

Depending on changes of signs in the sequence of $\lambda, m, \Lambda$ it has one, two or no solutions.

In Table 2 we illustrated the phase-portrait on $\varepsilon=0$ plane for a positive and a negative $\Lambda$, respectively for $n=3$.

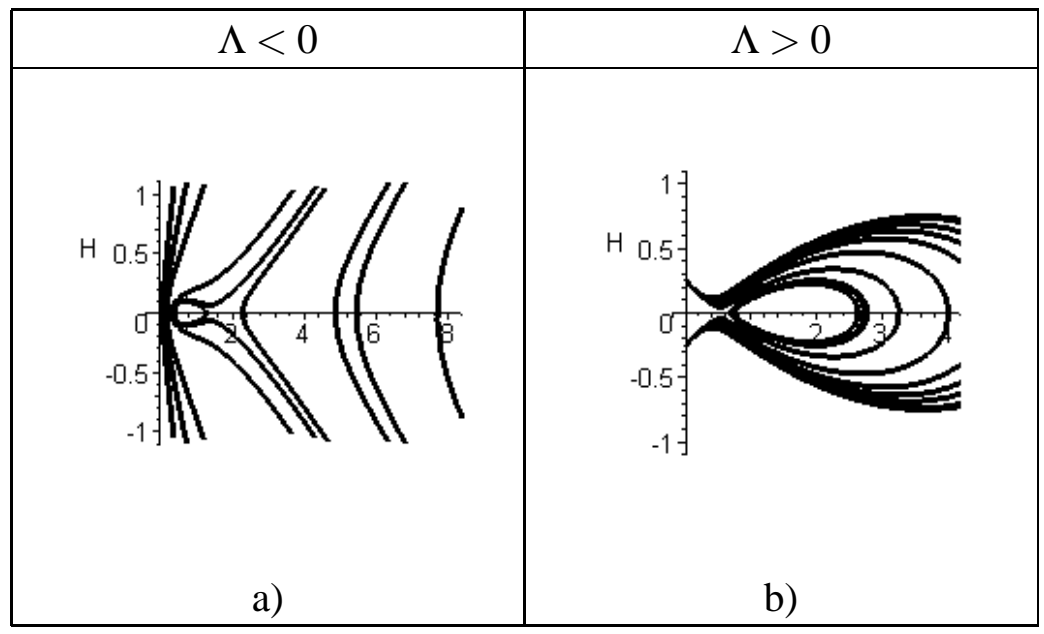

Table 2. Classification of qualitatively different types of evolution (phase portrait) on $\varepsilon=0$ plane for $n=3$ 
In Table 3 we have graphically illustrated the $H-v$ phase portrait on the $\varepsilon=0$ plane for different $\Lambda$. As it was mentioned earlier, here we deal with the multi-parametric system of ordinary nonlinear differential equation. In doing so we consider all possible variants independent to their physical validity. Therefore, we demonstrate the results obtained for a negative spinor mass $(m<$ $0)$.

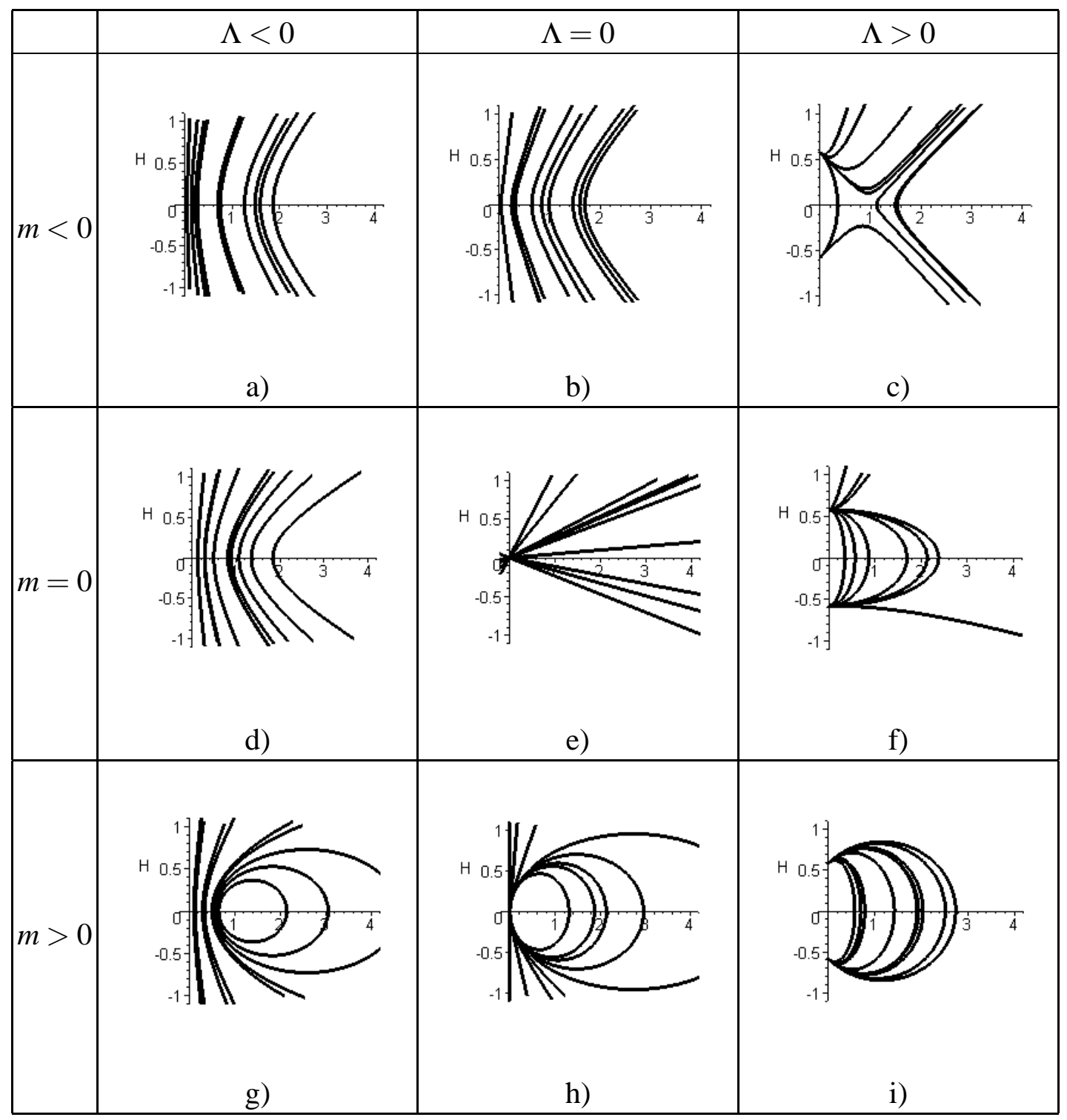

Table 3. Classification of qualitatively different types of evolution (phase portrait) on $\varepsilon=0$ plane for $n=2$

If the right hand side of (3.12) possesses two positive roots with $H$ being positive between them, then on the plane $\varepsilon=0$ there occurs closed cycle. It is obvious that there can be no more than three roots, hence there cannot be non-concentric cycles. As a result, near the plane $\varepsilon=0$ there might be cyclic oscillations.

The singular point around which the oscillation takes place has $H=0$, and therefore, the trajectory of oscillation partially passes in the region which is attractive to the plane $\varepsilon=0$ and partially 
in the region that is repulsive. In the long run in the repulsive region at some moment the growth of $\varepsilon$ becomes dominant. It results in the fact that $\varepsilon$ becomes infinity within a finite range of time.

\section{Invariants of evolution}

The system (3.1) in absence of viscosity, i.e., under $\eta=0$ and $\xi=0$ possesses the following first integrals

$$
\begin{aligned}
& F_{1}=\frac{\varepsilon}{v^{1+\zeta}} \\
& F_{2}=\frac{\left(H^{2}-\varepsilon-\Lambda-m v\right)}{v^{2}}+\lambda \frac{n-2}{n-3} v^{n-3}
\end{aligned}
$$

The first of them (3.14a) remains to be the first integral even after the introduction of bulk viscosity $\xi$. The second one, i.e., Eq. (3.14b) under $\xi \neq 0$ ceases to be the integral of motion. Nevertheless, the introduction of bulk viscosity during the course of time generates definite displacement of the surface given by the formula (3.14b), which allows one qualitatively, i.e., based only on the continuity, compile the representation about the possible ways of evolution.

\section{Qualitative analysis of the complete system}

Harnessing the Tables 2, 3 as well as 1, helps one to understand the 3D phase portrait leaning on the continuous dependence of the velocity fields of the coordinates $v, H, \varepsilon$ of phase space.

In order to cover the infinite phase space completely, it is mapped on coordinate parallelepiped with its axes being the the arc-tangent of the corresponding coordinates. The lower horizontal plane always represents the $\varepsilon=0$ plane.

It should be noted that the introduction of spinor field notably complicates the evolution of the system. Contrary to the system in absence of the spinor field, the initial condition with $H<0$ already does not prevent in many cases thanks to the evolution of volume scale entering the halfspace $H>0$ and thereupon, from the greater value of $H$ repeats the evolution, approaching to the $v=0$ plane and displaying the classification from the table 1 . In the vicinity of the borders $\varepsilon=0$ and $v=0$ the integral curves closely repeats the integral curves on the sides, each time at least to some extent.

The general property of all the cases is the fact that in the half-space $H>0$ the velocity vectors are directed to the $\varepsilon=0$ plane, while in the other half opposite to it. As a result all he invariant curves fall on $\varepsilon=0$, though not necessarily reach it.

In the Figs. 1 - 12 we have illustrated the the function inverse proportional to volume scale, i.e., $v(t)$ [Figs. 14/710], volume scale $\tau(t)$ [Figs. 215/811] and the phase portrait in the $v, H, \varepsilon$ space [Figs. 3619 12] , corresponding to the positions c,g,h, and i of the Table 2. In doing so we used the following values of the parameters: $\alpha=4, \beta=1, \zeta=0.5, A=1, B=1$ and $n=4$.The positions $\mathrm{h}$ and $\mathrm{i}$ correspond to the geometrically cyclic regime, but the case $\mathrm{h}$ possesses fixed point on cyclic integral curve, hence corresponds to the intermediate stage between periodic and non-periodic. The positions $\mathrm{c}$ and $\mathrm{g}$ correspond to the non-periodic evolution.

The bold black line in the 3D figures [Figs. 366912] corresponds to the the functions $v(t)$ and $\tau(t)$ presented in the preceding Figures [Figs. 1/4/7/10/2[5/8]11]. Figs. 1/2/3 and 10]11/12 correspond to the case $\mathrm{k}$ of Table 1, Fig. 45/6 to i and Fig. 7/89 to h, respectively.

The simplest case is illustrated in Figs. 10/1112. The integral curves beginning from anywhere falls into $v=0$. The other peculiarities of this curve are of no significance for the volume scale. 
In case of Fig. 12/3, there exists hyperbolic singular point on the $\varepsilon=0$ plane, so additional to the Fig. 10[1112 there exists curves tending to the side $v=\infty$.

The cyclic trajectories of Figs. 4516 beginning at the vicinity of $\varepsilon=0$ plane, gradually moving away, approach to the $\varepsilon=\infty$ plane, and the plane itself in the long run turns out to be attracting one with the edges $H= \pm \infty$.

The geometrically cyclic lines in Figs. 789 in the $\varepsilon=0$ plane contains fixed point, hence the behavior of the corresponding trajectory is very similar to the one given in Figs. 10]11112,

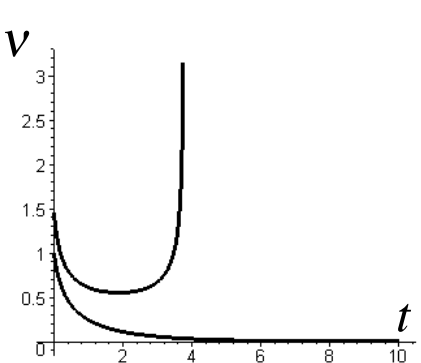

FIG. 1: Evolution of function inverse to volume scale

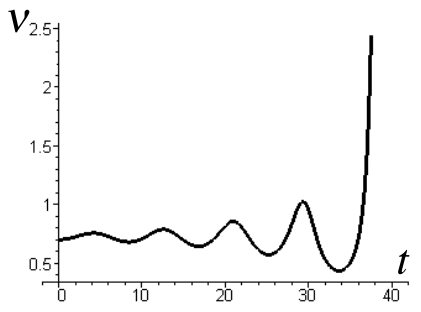

FIG. 4: Evolution of function inverse to volume scale $\tau$

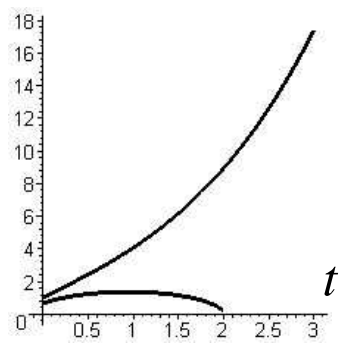

FIG. 2: Evolution of volume scale $v, H, \varepsilon$ space
FIG. 3: 3D view in

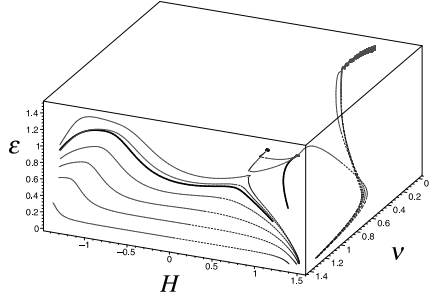

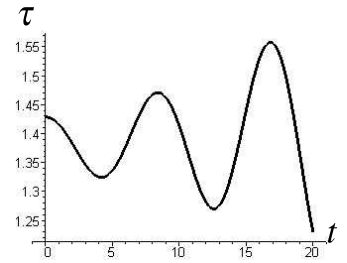

FIG. 5: Evolution of volume scale 


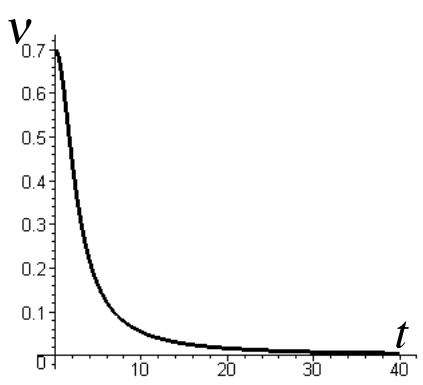

FIG. 7: Evolution of function inverse to volume scale

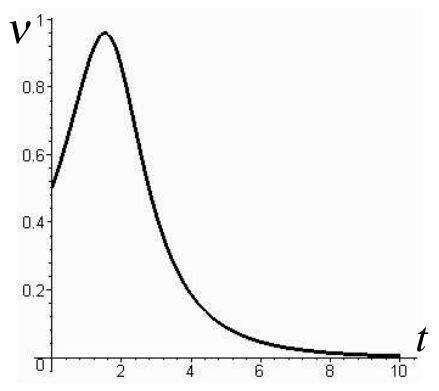

FIG. 10: Evolution of function inverse to volume scale

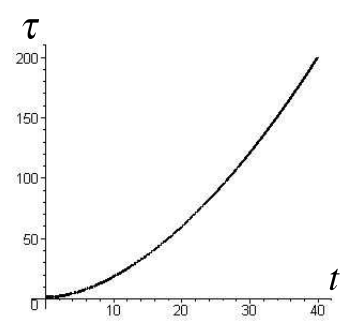

FIG. 8: Evolution of volume scale

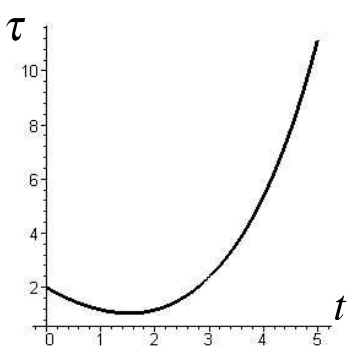

FIG. 11: Evolution of volume scale

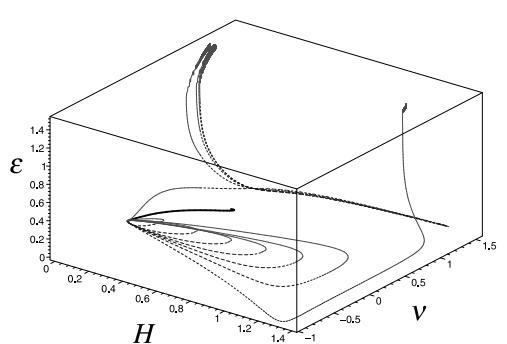

FIG. 9: 3D view in $v, H, \varepsilon$ space

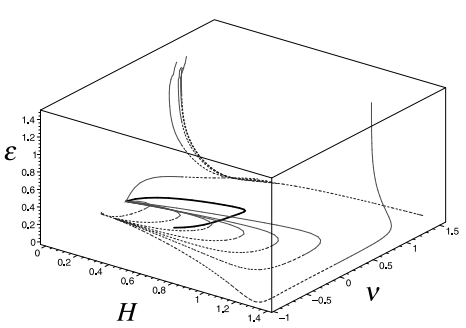

FIG. 12: 3D view in $v, H, \varepsilon$ space

independent to their physical validity and graphically presented the most distinguishable in our view results.

[1] Bijan Saha, Romanian Reports of Physics, 57(1), 7 (2005).

[2] Bijan Saha, Nonlinear spinor field in Bianchi type-I Universe filled with viscous fluid: numerical solutions [arXiv: gr-qc/0703085].

[3] W. Misner, Nature 214, 40 (1967).

[4] W. Misner, Astrophysical Journal 151, 431 (1968).

[5] S. Weinberg, Astrophysical Journal 168, 175 (1972).

[6] G.L. Murphy, Physical Review D 8, 4231 (1973).

[7] V.A. Belinski and I.M. Khalatnikov, Journal of Experimantal and Theoretical Physics 69, 401, (1975).

[8] B. Saha and G.N. Shikin, Journal of Mathematical Physics 38, 5305 (1997).

[9] B. Saha, and G.N. Shikin, General Relativity and Gravitation 29, 1099 (1997).

[10] Bijan Saha, Physical Review D 64,123501 (2001).

[11] Bijan Saha, Physics of Particles and Nuclei 37 Suppl. 1, S13-S44, (2006).

[12] Bijan Saha, Physical Review D 74, 124030, (2006).

[13] Ribas, M.O., Devecchi, F.P., and Kremer, G.M., Phys. Rev. D 72 (2005) 123502. 
[14] Bijan Saha, Modern Physics Letters A, 20(28), 2127 (2005).

[15] Bijan Saha and V. Rikhvitsky, Physica D 219, 168 (2006).

[16] T.W.B. Kibble, J. Math. Phys. 2, 212 (1961)

[17] V.B. Berestetski, E.M. Lifshitz and L.P. Pitaevski, Quantum Electrodynamics (Nauka, Moscow, 1989).

[18] K.C. Jacobs, Astrophysical Journal 153, 661 (1968).

[19] V.A. Zhelnorovich, Spinor theory and its application in physics and mechanics (Nauka, Moscow, 1982).

[20] D. Brill and J. Wheeler, Review of Modern Physics 29, 465 (1957).

[21] E Kamke, Differentialgleichungen losungsmethoden und losungen (Leipzig, 1957).

[22] Ya.B. Zeldovich and I.D. Novikov, Structure and evolution of the Universe (Nauka, Moscow, 1975).

[23] S.W. Hawking and R. Penrose, Proceedings of the Royal Society of London. Mathematical and physical sciences 314, 529 (1970).

[24] W. Heisenberg, Introduction to the unified field theory of elementary particles (Interscience Publ., London, 1966). 